\title{
Effective Connectivity in Response to Posture Changes in Elderly Subjects as Assessed Using Functional Near-Infrared Spectroscopy
}

\author{
Congcong Huo ${ }^{1}$, Ming Zhang ${ }^{2}$, Lingguo Bu ${ }^{1}$, Gongcheng $\mathrm{Xu}^{1}$, Ying $\mathrm{Liu}^{3,4}$, Zengyong $\mathrm{Li}^{3,4 *}$ \\ and Lingling Sun ${ }^{1 *}$
}

${ }^{1}$ Key Laboratory of High Efficiency and Clean Mechanical Manufacture, School of Mechanical Engineering, Shandong University, Jinan, China, ${ }^{2}$ Department of Biomedical Engineering, Faculty of Engineering, The Hong Kong Polytechnic University, Hong Kong, Hong Kong, ${ }^{3}$ Beijing Key Laboratory of Rehabilitation Technical Aids for Old-Age Disability, National Research Center for Rehabilitation Technical Aids Beijing, Beijing, China, ${ }^{4}$ Key Laboratory of Rehabilitation Aids Technology and System of the Ministry of Civil Affairs, Beijing, China

\section{OPEN ACCESS}

Edited by:

Stephane Perrey,

Université de Montpellier, France

Reviewed by:

Muthuraman Muthuraman Johannes-Gutenberg-University

Hospital, Germany

Noman Naseer,

Air University, Pakistan

*Correspondence:

Zengyong $\mathrm{Li}$

lizengyong@nrcrta.cn

Lingling Sun

sunl/@sdu.edu.cn

Received: 06 December 2017 Accepted: 28 February 2018

Published: 16 March 2018

Citation:

Huo C, Zhang M, Bu L, XU G, Liu Y, Li Z and Sun L (2018) Effective

Connectivity in Response to Posture

Changes in Elderly Subjects as Assessed Using Functional Near-Infrared Spectroscopy.

Front. Hum. Neurosci. 12:98. doi: 10.3389/fnhum.2018.00098
This study aims to assess the posture-related changes in frequency-specific effective connectivity (EC) in elderly subjects by coupling function measured using functional near-infrared spectroscopy (fNIRS). The fNIRS signals were continuously recorded from the bilateral prefrontal cortex (PFC), motor cortex (MC), and occipital lobe (OL) in 17 healthy elderly and 19 healthy young subjects during sitting and standing states. EC was calculated based on Dynamic Bayesian inference in one low frequency interval I: $0.052-0.145 \mathrm{~Hz}$ and one very low frequency interval II: $0.021-0.052 \mathrm{~Hz}$. Results show that in response to posture change, the coupling strength significantly increased in interval I of the young group from right PFC to MC $(p<0.05)$. Meanwhile, the coupling strength of the elderly group was significantly increased in interval II from the left PFC to right PFC $(p=0.008)$ and to left MC $(p=0.031)$ in the standing state as compared with that in the sitting state. Compared with that of the young group, the coupling strength of the elderly group was significantly decreased $(p<0.05)$ between the right PFC and left PFC in interval I and from PFC and OL to MC in interval II during the sitting state. The decreased EC in interval I was also positively correlated with cognitive scores in the elderly group. In addition, the coupling strength from MC to PFC in interval II during standing state was significantly increased in elderly subjects as compared with that in the young group. These results revealed the age-related changes in reorganization of interregional interactions for different postures. These findings may provide evidence of impaired cognitive function in the elderly and can deepen the understanding on age-related changes in neurovascular coupling.

Keywords: posture change, aging, effective connectivity, near-infrared spectroscopy, dynamic Bayesian inference

\section{INTRODUCTION}

Normal aging is related with decreased cognitive functions, including lack of attention, memory loss, and executive function decline (Hedden and Gabrieli, 2004; Kiyoka et al., 2013), which seriously affect their quality of life (Mitchell et al., 2010). Age-related cognitive decline is accompanied by the changes in neuronal activity and attenuation of functional interaction among 
different brain regions (Taniwaki et al., 2007; Spreng and Schacter, 2012). Maintaining a static standing posture is essential in executing daily activities. Analyzing of the age-related changes in brain network connections through different body postures allows an in-depth detection of the functional performance of neurovascular coupling mechanism of elderly people. However, the age-related changes in the reorganization of directed interregional interactions on the brain network under different posture states remain poorly understood.

Brain vasculature can respond to different conditions to regulate the blood flow. Regional cerebral blood flow (rCBF) must be allocated appropriately according to compartmentalized brain functions to meet different functional requirements. This process involves neurovascular coupling (Hotta, 2016). From sitting to standing states, the cardiovascular system maintains a stable rCBF to support sufficient levels of brain activity through myogenic, neurogenic, or metabolic mechanisms (Beek et al., 2008). These physiological mechanisms play a role in neurovascular coupling. Age-related functional deterioration of neurovascular coupling (Stefanova et al., 2013; Fabiani et al., 2014) contributes to the decline in high cortical functions, including cognition (Sorond et al., 2013) and motor coordination (Sorond et al., 2011). Hence, age-related deterioration may be reflected in the brain functional network based on neurovascular coupling. New strategies must be developed to determine the prognosis and monitor the cognitive decline with aging. This study on posture-dependent brain network provides an indepth understanding of the age-related changes in neurovascular coupling.

Functional near-infrared spectroscopy (fNIRS) is a noninvasive neuroimaging technology that is particularly sensitive to microvasculature (Obrig et al., 2000; Li et al., 2010; Sasai et al., 2012). The cerebral signals measured by fNIRS consist of endogenous systemic activity and the neurovascular coupling (Scholkmann et al., 2014). This method is used to monitor brain activities by measuring the relative regional changes in hemoglobin concentration and analyzing tight neurovascular coupling between neural activity and rCBF (Girouard and Iadecola, 2006; Kamran et al., 2015). Moreover, fNIRS are currently used to acquire brain signals for brain machine interface $(\mathrm{BCI})$ and have been popularized due to their safety and well-balanced spatial and temporal resolution (Naseer and Hong, 2015; Noori et al., 2017). Sasai reported that oscillation signals, which were simultaneously recorded from fNIRS and functional magnetic resonance imaging (fMRI), are well correlated (Sasai et al., 2012). In comparison with fMRI, fNIRS exhibited some advantages, such as relatively high temporal resolution $(10 \mathrm{~Hz})$ and insensitivity to movement artifacts. Hence, fNIRS is especially appropriate for examining brain function of the elderly in natural environments.

The brain is a complex network that includes multiple regions with specific functions. Information processing requires

Abbreviations: EC, Effective Connectivity; FC, Functional Connectivity; LPFC, Left Prefrontal Cortex; RPFC, Right Prefrontal Cortex; LMC, Left Motor Cortex; RMC, Right Motor Cortex; LOL, Left Occipital Lobe; ROL, Right Occipital Lobe; WT, Wavelet Transform; mCD, main coupling direction. functional dynamic interaction among segregated brain areas (Sporns and Kötter, 2004). One method of characterizing the interaction relationship is functional connectivity (FC), which describes such relationship as "statistical dependencies among distinct neurophysiological regions" (Biswal et al., 2010; Friston, 2011). Reconstructing dynamic human movement from a sequence of static body postures could increase the FC that is related to the motor cortex (MC) (Orgs et al., 2016). Our previous study revealed posture-related changes in the brain FC based on wavelet phase coherence in elderly subjects (Wang et al., 2016). However, analysis of FC does not involve inference on coupling among brain regions. The interactions among different brain areas are dynamic and directional, but the results cannot reveal the causal interaction and direction of influences in the brain network. To fully understand the neurovascular coupling interaction among brain regions, we adopted effective connectivity (EC) to investigate these interactions (Park and Friston, 2013). EC refers explicitly to "the causal influence one neural system exerts over another" and can characterize the dynamic causality of the brain network (Friston, 1994). Granger causality is a popular method for calculating EC in fMRI. However, this method can only infer the existence of causal effect but not the causal coupling per se (Park and Friston, 2013). Hence, the EC calculated by Granger causality is regarded as a measure of directed FC. By comparing with Granger causality, we based the Bayesian inference on a dynamical model that can infer the underlying causal mechanisms of the brain (Friston, 2011).

In this study, the prefrontal cortex (PFC), MC, and occipital lobe (OL) were covered by 36 channels to measure oscillation signals with fNIRS. PFC is essential for emotional control and plays an important role in planning complex cognitive behavior, decision making, and forming personal expressions (Miller and Cohen, 2001). PFC may also be involved in maintaining attention-demanding balance tasks (Mihara et al., 2008; Basso et al., 2014). MC is mainly responsible for coordination of sensory and motor functions (Hogenhout, 2013). OL is crucial in coordination of language, perception, and abstraction and is essential for processing visual information (Moran and Desimone, 1985; Corbetta et al., 1991; Astafiev et al., 2004). These regions may be involved in a differently coordinated fashion in response to posture changes.

In this study, we hypothesized that age-related changes exist in EC network in response to posture change. The directed coupling interactions among the brain regions were calculated based on a mode of coupled phase oscillator and Dynamical Bayesian inference (Tomislav et al., 2016), from which the influence exerted by one oscillator on another was determined, and the underlying causality was predicted. Analyzing the age-related changes in brain network connections through in different posture states may deepen our understanding of the basis of function deficits, which may emerge during normal (non-pathological) aging. This study offers a new method of accessing and preventing cognitive loss and detecting the functional performance of neurovascular coupling in the elderly. 


\section{MATERIALS AND METHODS}

\section{Participants}

Thirty-eight healthy volunteers were recruited from the university and categorized in two age groups: the first group (elderly group) was composed of 18 elderly subjects (all righthanded; 5 females, 13 males), the second group (young group) was composed of 20 healthy young subjects (all right-handed; 7 females, 13 males). The subjects were screened to ensure that they did not have a history of hypertension, abnormal heart, kidney, liver, and lung functions. In this study, hypertension was defined as systolic blood pressure $\geq 140 \mathrm{~mm} \mathrm{Hg}$ or diastolic blood pressure $\geq 90 \mathrm{~mm} \mathrm{Hg}$ (Jones et al., 2003). All subjects were free from psychiatric disorders or neurological illness and were not treated with any psychotropic medication. All participants provided written informed consent before participating in this study. The experimental procedure was approved by the Human Ethics Committee of National Research Center for Rehabilitation Technical Aids and was in accordance with the ethical standards specified by the Helsinki Declaration of 1975 (revised in 2008).

\section{Data Acquisition}

Prior to the experiment, we recorded basic information of our subjects, including age, sex, height, weight, and their individual the Mini Mental State Examination (MMSE) scores. Table 1 shows the characteristics of the participants. fNIRS measurements were conducted with a multi-channel tissue oxygenation monitor (NirScan Danyang Huichuang Medical Equipment CO. Ltd) with three wavelengths (755, 808, and $855 \mathrm{~nm}$ ) to measure the change in regional blood volume. The reflection of the infrared light was tested by source probes placed on the scalp $30 \mathrm{~mm}$ away from the detector probes. We initially set all differential path-length factors to 6.0. Tissue absorbency measurements were recorded and converted into optical density units, and the concentration changes in $\mathrm{HbO} 2$ (Delta [O2Hb]) were calculated based on the modified Beer-Lambert law (Teng et al., 2006). The sampling rate was $10 \mathrm{~Hz}$. Recordings were derived from 36 channels contributed by sources and detectors (Figure 1), which were positioned over the left PFC (LPFC), right PFC (RPFC), left MC (LMC), right MC (RMC), left OL (LOL), and right OL (ROL) in accordance with the International 10/10 System. Each channel arrangement was accurately corresponded to the 10/10 electrode positions according to different head sizes by using the calibration function of the instrument and the corresponding template.

The probes were covered with a loose-fitting black cloth to minimize interference of ambient light on optical signals. Measurements were performed in a quiet room with dim light at room temperature. The subjects were instructed to maintain a comfortable sitting position, remain still, relax with eyes closed, and avoid drastic movements. Signal recordings were collected at the sitting state for $15 \mathrm{~min}$. The subjects were reminded to stay awake and not fall asleep. After collecting the sitting state data, we required the subjects to stand up and maintain their balance. Signal recordings were collected at the standing state for $10 \mathrm{~min}$. After completion of the experiment, a questionnaire survey was
TABLE 1 | Demographic information.

\begin{tabular}{lccc}
\hline Parameter & Old $(\boldsymbol{n}=\mathbf{1 7})$ & Young $(\boldsymbol{n}=\mathbf{1 9 )}$ & $\boldsymbol{p}$ for difference \\
\hline Age (years) & $69.4(9.6)$ & $24.1(1.9)$ & $0.000^{\star}$ \\
BMl & $19.2(3.2)$ & $18.5(4.2)$ & 0.584 \\
MMSE & $27.5(1.5)$ & $29.3(0.6)$ & $0.000^{\star}$ \\
\hline
\end{tabular}

Values are presented as means and standard deviations. BMI, Body Mass Index; MMSE, Mini-mental state examination. *Significant group differences $p<0.05$.

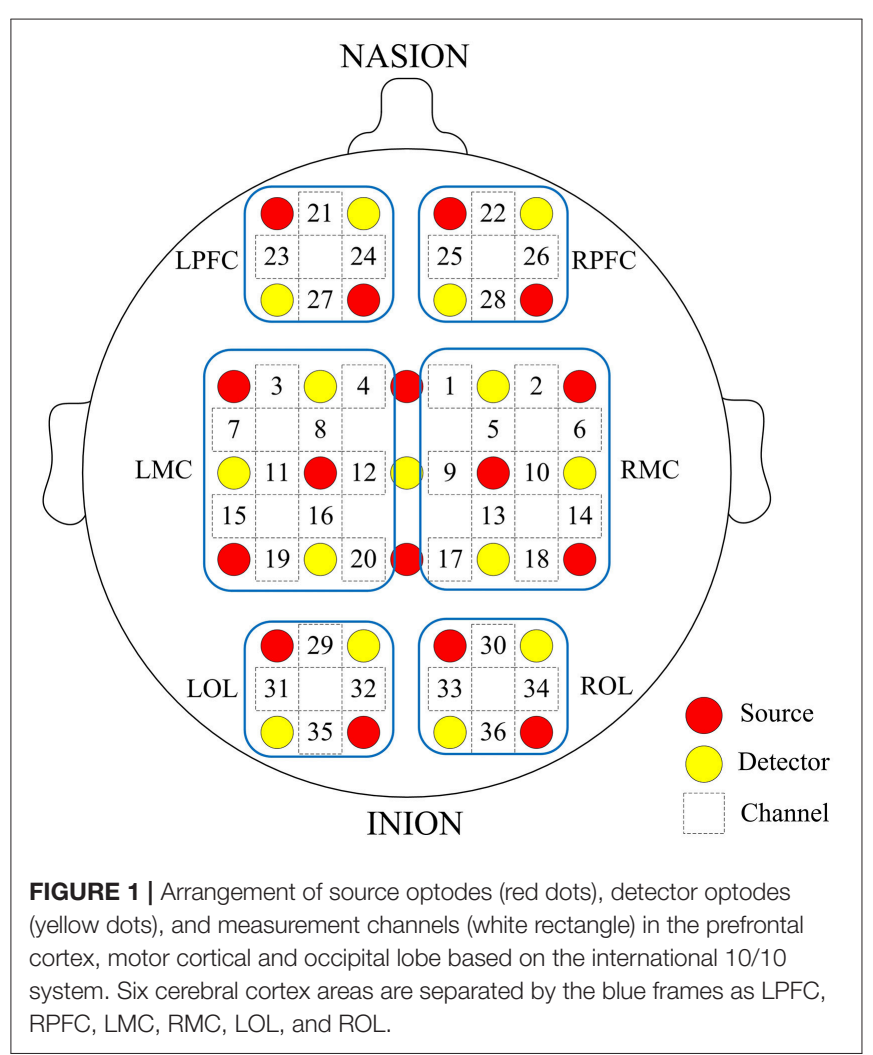

conducted to examine whether or not the subjects were asleep during resting state, and to ensure quality of the experiment.

\section{Data Pre-processing}

The pre-processing method for fNIRS data, which has been described in detail in previous studies (Han et al., 2014; Xu et al., 2017), was conducted as follows. First, moving average method was applied to remove the noise-like abrupt spikes caused by movement artifacts or background light. This method was based on moving standard and spine interpolation routines. Second, to remove uncorrelated noise components (above $2 \mathrm{~Hz}$ ) and low-frequency variations (below $0.005 \mathrm{~Hz}$ ), we employed a Butterworth band-pass filter to obtain filtered signals of $0.005-2 \mathrm{~Hz}$. One young subject and one elderly subject were excluded because of excessive head artifact interference in their signals. 


\section{Wavelet Transform (WT)}

WT is a method that transforms signals from the time domain to the time-frequency domain by using frequencyadjustable filtering window and obtains a three-dimensional map containing time-frequency-amplitude information. The method of WT has been described previously in detail (Han et al., 2014). At a specific frequency $f$ and time point $t_{n}$, complex wavelet coefficients obtained by WT are defined as:

$$
w_{k}\left(t_{n}\right)=W_{k}\left(f, t_{n}\right) \cdot e^{i \emptyset_{k}\left(f, t_{n}\right)}=a_{k}\left(f, t_{n}\right)+i b_{k}\left(f, t_{n}\right)
$$

Wavelet instantaneous phase is defined as $\Phi_{k}\left(f, t_{n}\right)$ :

$$
\Phi_{k}\left(f, t_{n}\right)=\arctan \left(b_{k}\left(f, t_{n}\right) / a_{k}\left(f, t_{n}\right)\right)
$$

Through WT, the phase information was extracted from each filtered time series to build a series of coupled-phase-oscillator models.

These oscillation signals measured by fNIRS implied different physiological meanings at different frequency intervals, reflecting myogenic, neurogenic, or metabolic regulation of microvascular blood flow (Han et al., 2014; Vermeij et al., 2014). Two major frequency-specific oscillator intervals have been distinguished by WT in the intervals as follows: I-myogenic activity (0.052$0.145 \mathrm{~Hz}$ ) and II-neurogenic activity $(0.021-0.052 \mathrm{~Hz}$ ) (Shiogai et al., 2010).

\section{Coupling Strength and Direction}

Bayesian theory is based on prior knowledge used to estimate current or posterior model parameters or uses the acquired knowledge to improve the reasoning (Stankovski et al., 2014).

$$
p_{\chi}(M \mid \chi)=\frac{\zeta(\chi \mid M) p_{\text {prior }}(M)}{\int \zeta(\chi \mid M) p_{\text {prior }}(M) d M}
$$

The properties of a network of N-coupled periodic oscillators can be analyzed based on its phase dynamics. A system of $\mathrm{N}$ stochastic differential equations varying with time was established according to the original signal phase information obtained by WT (Stankovski et al., 2014):

$$
\Phi_{k}(t)=w_{k}(t)+q_{k}\left(\Phi_{1}, \Phi_{2}, \ldots, \Phi_{n}, t\right)+\xi_{k}(t)
$$

Where $k=1, \cdots, N . N$ is the number of oscillators in the network; in this paper, $N=2 ; \omega_{k}(t)$ is defined as the parameter for the natural frequency; the deterministic base function $q_{k}$ describes the interacting dynamics of the coupled phase oscillators $\Phi_{1 \ldots N}$; and $\xi_{k}$ is considered as Gaussian white noise. The deterministic periodic part of the differential equation (4) for each oscillator is decomposed into a sum of base functions $\Phi_{k}=\exp \left[l\left(k_{1} \varphi_{1}+k_{2} \varphi_{2}+\ldots+k_{N} \varphi_{N}\right)\right]$ modulated by parameter $c_{b}^{(k)}$ varying with time by using Fourier approximation (Stankovski et al., 2014):

$$
\dot{\Phi}_{k}(t)=\sum_{b=-B}^{B} c_{b}{ }^{(k)} \phi_{b}\left(\Phi_{1}, \Phi_{2}, \cdots, \Phi_{n}\right)+\xi_{k}(t)
$$

The deterministic part $c_{b}^{(k)}$ is decomposed into the sum of the partial contributions of different orders of coupling to isolate the different orders of the network coupling:

$$
\begin{aligned}
& \dot{\Phi}_{k}(t)=c_{0}^{(k)}+\sum_{b=-B}^{B} c_{b}^{\prime(k: l)} \phi_{b}\left(\Phi_{l}\right)+\sum_{b=-B}^{B} c_{b}{ }^{\prime \prime(k: l, m)} \phi_{b}\left(\Phi_{l}, \Phi_{m}\right) \\
& +\sum_{b=-B}^{B} c_{b}^{\prime \prime \prime}(k: l, m, n) \phi_{b}\left(\Phi_{l}, \Phi_{m}, \Phi_{n}\right)+\cdots+\xi_{k}(t)
\end{aligned}
$$

Where $k \neq 0 ; l, m, n=1, \ldots N$ and $l$ nem nen; $c_{b}^{(k)}$ the vector of coefficients of different orders of the coupling. The model's Fourier components act as base function for the Dynamical Bayesian inference and were used to evaluate the parameters $c_{b}^{(k)}$. We recursively calculated the parameters $c_{b}^{(k)}$ using the equations listed in the paper (Stankovski et al., 2014) based on Bayesian theory. We used the inferred $C$ to characterize coupling mechanisms in the network, which contained information on coupling strength and direction. The coupling strength is used to quantify the coupling amplitude, the Euclidean norm of inferred coupling coefficient matrix from oscillators $\phi_{i}$ and $\phi_{j}$.

In this study, we established coupling functions based on coupled-phase-oscillator model in a pairwise manner and utilized Dynamical Bayesian theory to infer directed coupling interactions for all possible pairs of channels. For the two channels, $i$ and $j$, we calculated the coupling influence that $i$ exerted on $j\left(c_{i \rightarrow j}\right)$ and $j$ on $i\left(c_{j \rightarrow i}\right)$ in each frequency interval and posture state of each subject. Meanwhile, the value of $c_{i \rightarrow j}$ is defined as the coupling strength from channel $i$ to $j$. A total of 1,260 frequency-specific directed coupling interactions were derived from all possible pairs of 36 channel signals for each subject and specific posture state. We used surrogate data testing to validate coupling interaction results. A total of 100 amplitude-adjusted Fourier transform (AAFT) surrogate signals were generated for every channel by shuffling the phases of the original time series to create new time series with the same means, variances, and autocorrelation functions as the original sequences but without their phase relations (Bernjak et al., 2012; Tan et al., 2016). The directed coupling interactions were derived from all possible pairs of the 100 surrogate signals for every two channels based on coupled-phase-oscillator model and dynamical Bayesian theory. We obtained the average of all coupling measurements calculated from these surrogate realizations of the signals. The directed coupling strength of the experimental signals was considered significant if the value was 2 standard deviations (SD) above the surrogate means. The coupling strength from the test denoted significant interaction. Results of the directed coupling interaction include coupling strength and direction. EC is derived from the concept of directed coupling interaction, which was based on a specific model of causal dynamics, to determine the causal influence that one oscillator exerts on another (Park and Friston, 2013). For each subject, the coupling interactions among all possible pairs of 36 channels were computed and tested by surrogate measurements for the specific posture state and frequency intervals. 


\section{Posture-Dependent Group-Averaged EC Network}

To construct the EC network at group level, we must test the existence of a connection between channels. Based on the individual significant connectivity, a connection at group level was considered when the connections between two channels exist in over $75 \%$ of the subjects. For group EC, the strength of each connection was the average coupling strength between two channels. The significant interactions of EC network are shown for each group and posture state.

\section{Main Coupling Direction Among the Six Brain Regions}

When the value of $C_{i \rightarrow j}$ exceeded $C_{j \rightarrow i}$, we defined $i \rightarrow j$ as the main coupling direction (mCD) between oscillator $\phi_{i}$ and oscillator $\phi_{j}$. For any individual subject, every significant interaction of all possible pairs of channels between two brain regions includes a $\mathrm{mCD}$, either from region 1 to region 2 or from region 2 to region 1 . Binomial tests were performed on the mCDs of significant interactions between each two brain regions to determine significant difference. A significant difference indicated the presence of $\mathrm{mCD}$, which plays a dominant role in the coupling function between the two brain regions; otherwise, bidirectional coupling between the two brain regions was considered.

\section{EC Among the Six Brain Regions}

This paper aimed to analyze the interaction among brain regions. For each subject, the coupling strengths of significant interactions between all possible pairs of 36 channels were averaged as over $A_{6}^{2}=30$ directed interregional connection types, thus corresponding to mutual interactions among the six regions. The channel distributions covered by each brain region are displayed in Figure 1. The $30 \mathrm{EC}$ values were obtained from each subject by averaging the coupling strength of channels between two brain regions for specific frequency interval and posture state.

\section{Correlation Analysis Between Interregional EC and Cognitive Performance}

To identify the association between the varying causal interactions and the cognitive performance of the elderly, we extracted and correlated the interregional coupling strength with the MMSE scores using Pearson's correlative analysis.

\section{Statistical Analysis}

Analysis of significant differences in the characteristics between young and elderly subjects was conducted using $t$-test for means and SD. Chi-square test was performed to evaluate the significant difference in sex percentages. Binomial-tests were performed on the mCDs of significant channel pairs between the two brain regions to determine the coupling direction of the inter-regions. Given the non-normal distribution of data, we conducted Kruskal-Wallis to test the significance level of frequency-specific EC of 30 connection types among different groups and conditions. The association between the interregional EC and cognitive performance was explored using Pearson correlation coefficients. A difference of $p<0.05$ was considered statistically significant.

\section{RESULTS}

\section{Demographic Data}

Participant characteristics are shown in Table 1. The two groups has similar body mass index (BMI) nut significantly different MMSE scores $(p<0.0001)$.

\section{Posture-Related Changes in EC Network}

Figures 2, 3 show the EC network of the 36 channels at the group level for specific frequency interval and posture states of the elderly group and young group. Different channel distributions represented different brain regions as displayed in the diagrams. Posture-related changes were observed in the EC network in different frequency intervals. A matrix box with color indicates a significant EC value between two channels, whereas the white matrix lattice represents no significant connection. Different colors represent different values of coupling strength.

\section{Age-Related Changes in mCD Between the Brain Regions}

In this study, we calculated the interregional EC among the six brain regions: LPFC, RPFC, LMC, RMC, LOL, and ROL. The $\mathrm{mCDs}$ among the brain regions are either bidirectional or unidirectional. Bidirectional connection between the two brain regions illustrates absence of dominant functional source of coupling. Thus, the two brain regions regulate each other. On the contrary, unidirectional connection indicates that one of the two brain regions serves as the predominant functional source of coupling and plays a major regulatory role, whereas the other region acts as the target of regulation. The reverse of the $\mathrm{mCD}$ is regarded as the feedback effect in this study.

Figures 4, 5 illustrate the $\mathrm{mCD}$ among the six brain regions in both groups and conditions. In interval I (Figure 4), from sitting to standing states, the coupling direction between the LMC and RPFC, LMC and RMC, and LOL and ROL in the elderly was transformed from bidirectional to unidirectional connection. This finding implies that MC played a stronger regulatory role in the standing state among the regions of the brain. In the young group, posture-related changes in $\mathrm{mCD}$ illustrated the regulation exerted by RPFC and ROL on RMC. This effect was increased in the standing state. The most predominant finding in this interval includes age-related changes in $\mathrm{mCD}$ among the brain regions. The $\mathrm{mCD}$ in the elderly was mostly observed from $\mathrm{MC}$ to $\mathrm{PFC}$ and to $\mathrm{OL}$, indicating that $\mathrm{MC}$ played a main regulatory role in the brain network of the elderly. However, the connections between PFC and OL were bidirectional in the elderly but unidirectional in the young group (from PFC to $\mathrm{OL})$. In summary, these results illustrated the occurrence of agerelated deterioration in the regulation function of PFC, especially for long-distance connections. Nonetheless, the MC in the elderly group plays a major regulatory role to compensate for the decline regulation function of PFC.

Figure 5 shows the posture-related changes in the $\mathrm{mCD}$ in interval II. In general, regulation from OL to $\mathrm{MC}$ and from PFC to $\mathrm{OL}$ increased in response to posture changes in both groups. In the sitting state, the $\mathrm{mCD}$ is from LMC to ROL in the elderly but from ROL to LMC in the young group. 

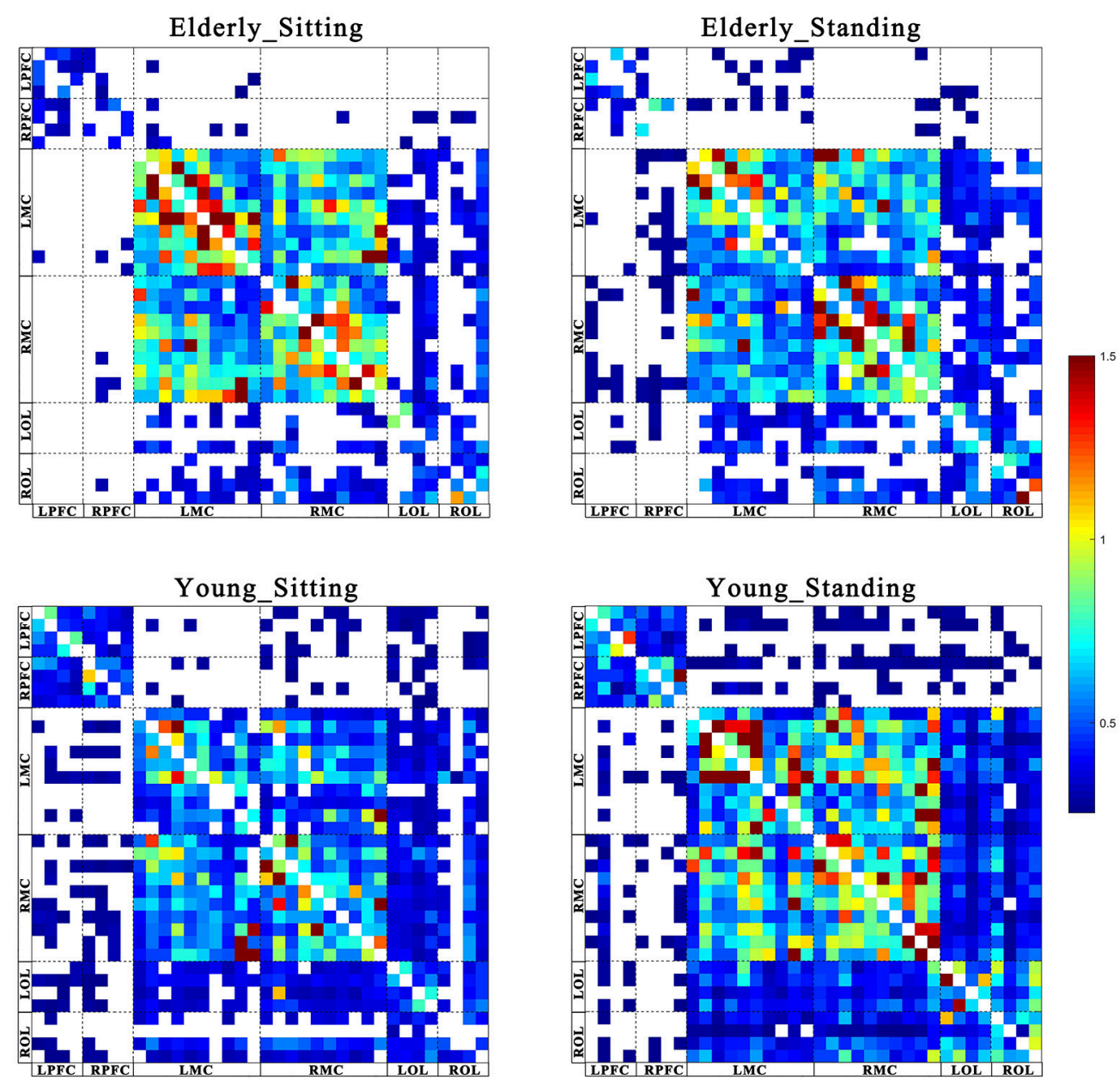

FIGURE 2 | Posture-related changes in effective connectivity network in frequency interval I. The frequency-specific asymmetric mean coupling strength matrix of two groups in different conditions (where 36 is the number of channels). The coupling direction between the channels is indicated from the row to the column. Different channels correspond to brain regions, Channel 1-4 (LPFC), Channel 5-8 (RPFC), Channel 9-18(LMC), Channel 19-28 (RMC), Channel 29-32 (LOL), and Channel 33-36 (ROL).

\section{Age-Related Changes in EC Under Different Posture States}

Coupling strengths of the significant connection between channel pairs were averaged to form 30 directed interregional EC types among the six brain regions. Frequency-specific connectivity was analyzed in both groups and both posture conditions to reveal the age-related alterations in EC from sitting to standing states. Figures 6, 7 show the significant changes in EC values among the six brain regions in both groups and conditions.

\section{Posture-Related Changes in EC}

From sitting to standing state, the coupling strengths of RPFC to $\mathrm{LMC}(p=0.03)$ and $\mathrm{RPFC}$ to $\mathrm{RMC}(p=0.02)$ (represented by $\mathrm{RPFC} \rightarrow \mathrm{LMC}, \mathrm{RPFC} \rightarrow \mathrm{RMC}$ ) were significantly increased in interval I (Figure 6) of the young group. By contrast, the coupling strength significantly increased from LPFC to RPFC $(p=0.008)$ and from LPFC to LMC ( $p=0.031)$ (represented by LPFC $\rightarrow$
$\mathrm{RPFC}, \mathrm{LPFC} \rightarrow$ LMC) in interval II (Figure 7) of the elderly group.

\section{Age-Related Changes in EC}

Compared with the young group, in the sitting state, the elderly group had significantly decreased EC as noted in $\mathrm{LPFC} \rightarrow \operatorname{RPFC}(p=0.023)$ and $\mathrm{RPFC} \rightarrow \operatorname{LPFC}(p=0.044)$ in interval I (Figure 6); and $\mathrm{LPFC} \rightarrow \mathrm{RPFC}(p=0.028)$, $\mathrm{LPFC} \rightarrow \mathrm{LMC}(p=0.02), \mathrm{RPFC} \rightarrow \mathrm{LMC}(p=0.042), \mathrm{LOL} \rightarrow \mathrm{LMC}$ $(p=0.004), \mathrm{ROL} \rightarrow \mathrm{LMC}(p=0.014), \mathrm{ROL} \rightarrow \mathrm{RMC}(p=0.012)$, and $\mathrm{ROL} \rightarrow \mathrm{RMC}(p=0.022)$ in interval II (Figure 7). Compared with the young group, the elderly group in the standing state had significantly increased EC of LMC $\rightarrow$ LPFC $(p=0.023)$, $\mathrm{LMC} \rightarrow \operatorname{RPFC}(p=0.025), \mathrm{RMC} \rightarrow \mathrm{LPFC}(p=0.022)$, and $\mathrm{RMC} \rightarrow \mathrm{RPFC}(p=0.009)$ in interval II (Figure 7). According to the results of $\mathrm{mCD}$ among the brain regions, the connections from MC to PFC in interval II of the elderly group in standing state had significantly higher coupling strength than those of 

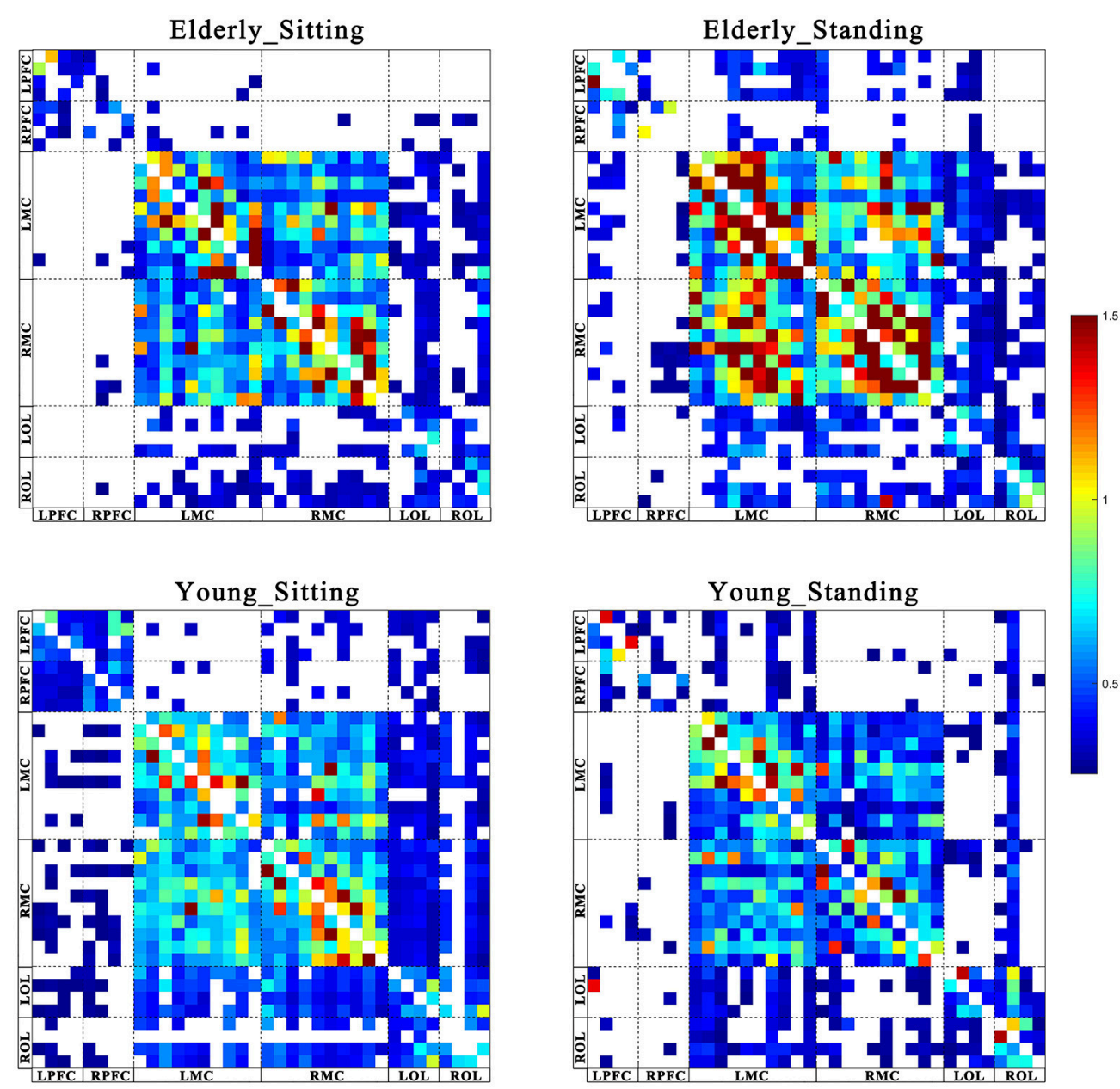

FIGURE 3 | Posture-related changes in effective connectivity network in frequency interval II. The frequency-specific asymmetric mean coupling strength matrix of two groups in different conditions (where 36 is the number of channels). The coupling direction between the channels is indicated from the row to the column. Different channels correspond to brain regions, Channel 1-4 (LPFC), Channel 5-8 (RPFC), Channel 9-18(LMC), Channel 19-28 (RMC), Channel 29-32 (LOL), and Channel 33-36 (ROL).

the young group. This finding corresponded to the feedback connections in the elderly group.

\section{Correlations Between Interregional EC and Cognitive Performance}

We found that the interregional causal interactions were correlated with MMSE scores in the elderly (Table 2). The MMSE scores were significantly and positively related with the directed coupling strength of LPFC $\rightarrow$ RPFC $(r=0.689, p=0.002)$, $\mathrm{RPFC} \rightarrow \mathrm{LPFC}(r=0.679, p=0.003), \mathrm{RPFC} \rightarrow \mathrm{LMC}(r=0.487$, $p=0.047), \mathrm{LMC} \rightarrow \mathrm{RPFC}(r=0.486, p=0.048), \mathrm{RPFC} \rightarrow \mathrm{RMC}$ $(r=0.531, p=0.028), \mathrm{RPFC} \rightarrow$ LOL $(r=0.505, p=0.039)$, $\mathrm{RPFC} \rightarrow \mathrm{ROL}(r=0.489, p=0.047)$ in interval I.

\section{DISCUSSION}

NIRS signals recorded on the head consist of endogenous systemic activity and neurovascular coupling (Scholkmann et al.,
2014). Oscillations in NIRS at different frequency intervals may reflect different neurovascular couplings and systemic regulation mechanisms. These regulatory mechanisms are considered working together to maintain a relatively stable rCBF at different states. The main findings of this study are the age-related alterations in EC in response to posture changes. From sitting to standing state, the influence of RPFC on MC significantly increased in interval I for the young group. However, the effect of LPFC on RPFC and LMC significantly increased in interval II for the elderly group. Compared with the young group, the elderly subjects had significantly decreased interregional coupling strength in intervals I and II in their sitting state. The decreased connectivity between LPFC and RPFC in interval I was also positively correlated with MMSE scores of the elderly subjects. The EC from MC to PFC in interval II was increased in elderly subjects in standing state, as compared with that in the young subjects. These findings revealed the age-related changes in reorganization of interregional interactions under 
A

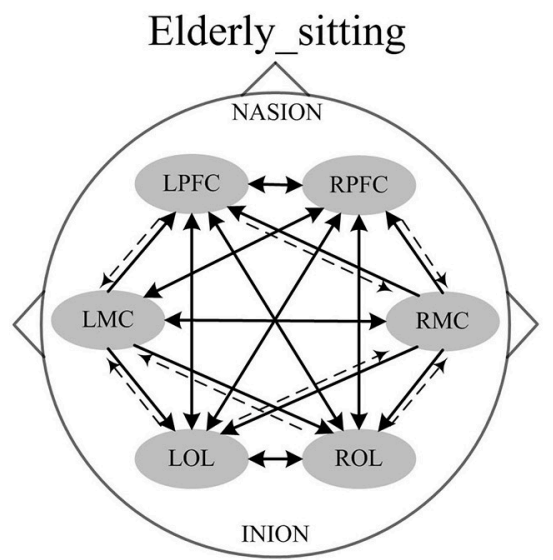

C

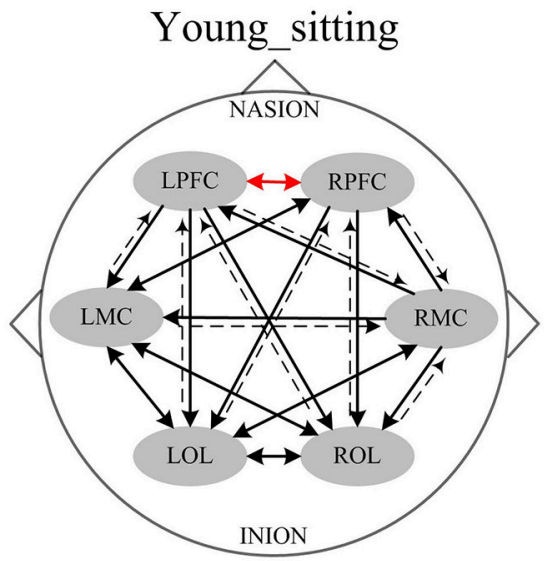

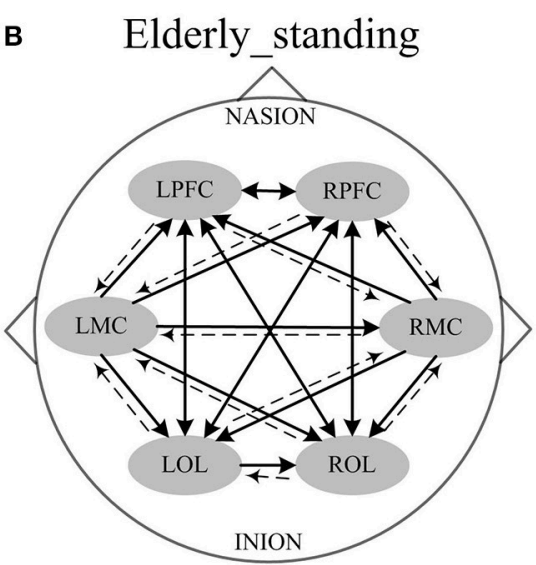

D Young_standing

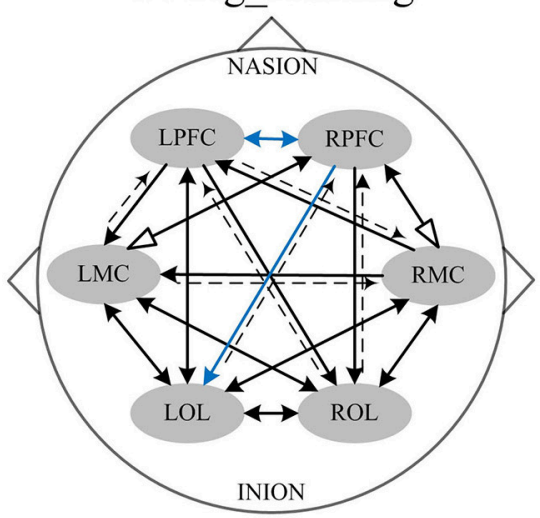

$\longleftrightarrow$ Bidirectional connection

$\longrightarrow$ Main coupling direction

$\rightarrow-\rightarrow$ Feedback effect (Non main coupling direction)

Significantly increased coupling strength in standing state compared with the sitting state

Main coupling direction connection that coupling strength was significantly higher as compared between the elderly and young groups in sitting state

Main coupling direction connection that coupling strength was significantly higher as compared between the elderly and young groups in standing state

FIGURE 4 | Comparison of the main coupling direction in interval I in the effective connectivity network among the six brain regions. The arrow points to the direction of the coupling interaction. Unidirectional arrows and bidirectional arrows are present between the two brain regions. (A) Elderly_sitting state,

(B) Elderly_standing state, (C) Young_sitting state, (D) Young_standing state.

different posture states and highlight the alterations of healthy aging in neurovascular coupling functions. These results may provide new insights into the development of new strategies to monitor cognitive loss and detect the functional performance of neurovascular coupling mechanism in the elderly.

The brain network is a coordinated system of specific brain regions working together to promote specific cognitive functions (Andrewshanna et al., 2007; Meunier et al., 2009; Wen et al., 2011). Structural, functional, and directional organizations of complex brain networks serve as basis for the realization of various brain functions. The interactions among the brain regions are different in various posture states. In this study, EC was calculated from neurovascular coupling signals based on a model of coupled phase oscillators and Dynamical Bayesian inference. The findings enabled us to estimate the coupling strength, $\mathrm{mCD}$ and underlying causality among brain regions in the sitting and standing states. Age-related alterations in the brain network in different posture states were analyzed according to the physiological meanings of oscillation signals in different frequency intervals (Li et al., 2013a; Han et al., 2014; Bu et al., 2016). 
A

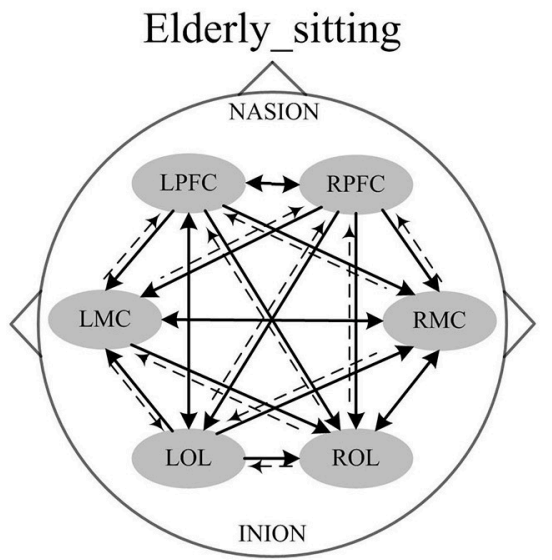

C

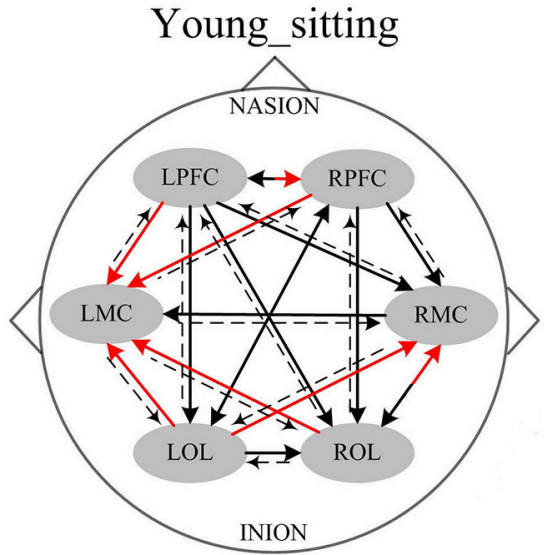

B Elderly_standing

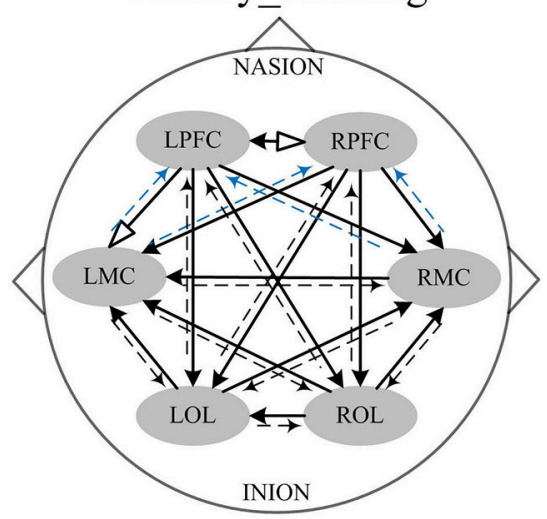

D Young_standing

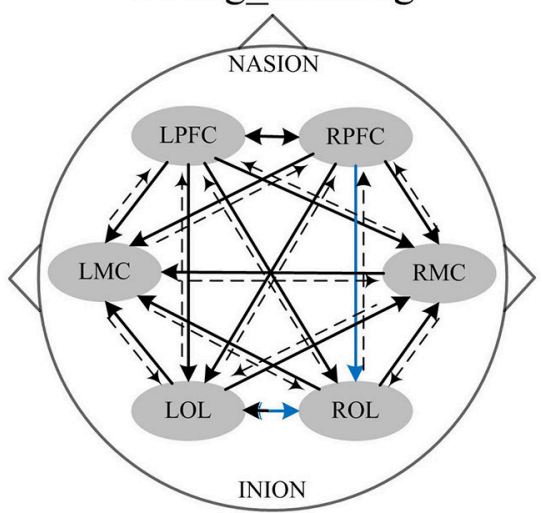

$\longleftrightarrow$ Bidirectional connection

$\longrightarrow$ Main coupling direction

$\rightarrow-\rightarrow$ Feedback effect (Non main coupling direction)

Significantly increased coupling strength in standing state compared with the sitting state

Main coupling direction connection that coupling strength was significantly higher as compared between the elderly and young groups in sitting state

Main coupling direction connection that coupling strength was significantly higher as compared between the elderly and young groups in standing state

Feedback connection that coupling strength was significantly higher as compared between the elderly and young groups in standing state

FIGURE 5 | Comparison of the main coupling direction in interval II in the effective connectivity network among the six brain regions. The arrow points to the direction of the coupling interaction. Unidirectional arrows and bidirectional arrows are present between the two brain regions. (A) Elderly_sitting state, (B) Elderly_standing state, (C) Young_sitting state, (D) Young_standing state.

The results showed significant age-related differences in EC in response to posture changes. Coupling strength analysis in interval II (Figure 7) indicated that the EC in elderly was significantly affected by posture. However, the same result was not observed in the young group. Within the brain, the cerebral oscillations in interval II were suggested as locally neurogenic activities originating from vascular reactions of neurogenic origin (Shiogai et al., 2010; Li et al., 2013b). Brain vasculature can adjust blood flow in response to local conditions. Neurogenic control of cerebral blood vessels is innervated by the surrounding vasoactive nerves, that is., sympathetic, parasympathetic and somatic sensory nerves on the brain surface, and is sufficient for regulating overall blood flow to the brain (Hotta, 2016). Sustained activity of the autonomous nervous system maintains the basal level of blood vessel contraction by releasing substances that affect smooth muscle activity. Hemodynamic parameters 
Old_Sitting VS Young_Sitting

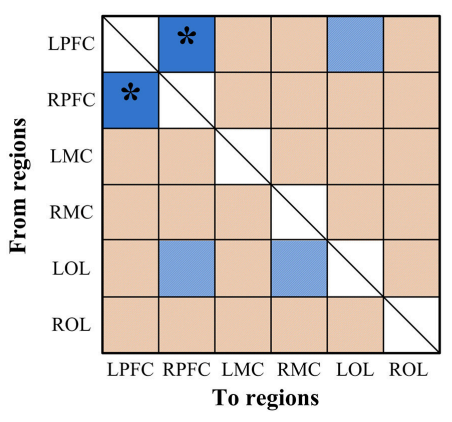

Young_Sitting VS Young_Standing

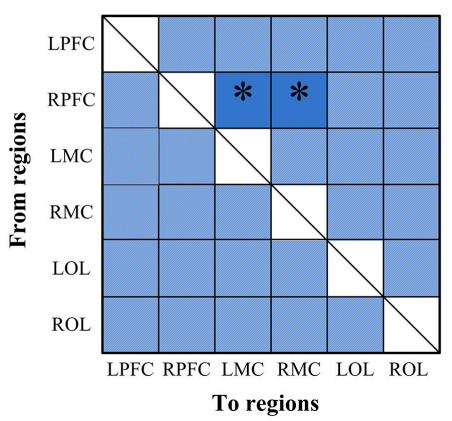

Old_Sitting VS Old_Standing

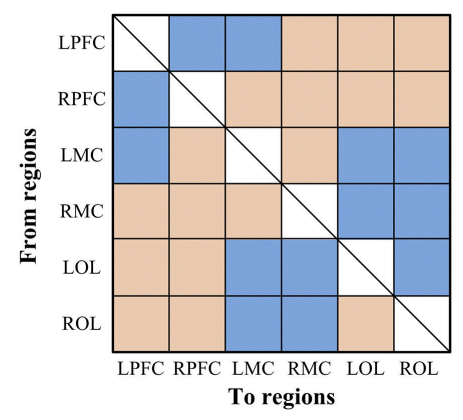

Old_Standing VS Young_Standing

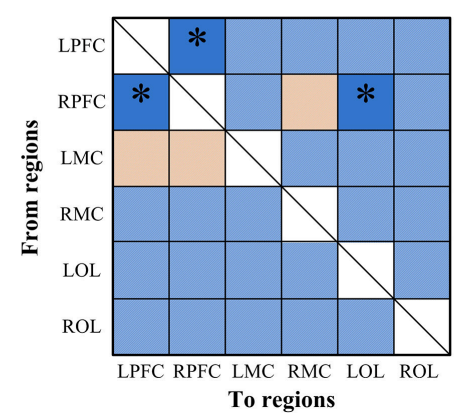

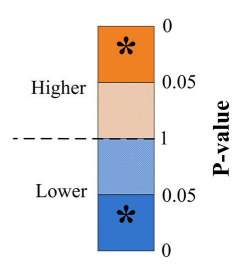

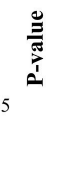

FIGURE 6 | Significance matrices representing significant difference between each two groups in EC among the six brain regions in interval I. In every two groups of contrast, the warm color represents that the former is greater than the latter, and the bright color with *represents a significance that is greater than the latter. Cold color represents that the former is less than the latter, and the darker cold color with *represents significance lower than the latter.

are closely regulated through tight neurogenic innervations and under partially autonomic control in interval II (Zhang et al., 2002; Shiogai et al., 2010). In the elderly group, causal influence from LPFC to RPFC and to LMC was significantly stronger in the standing state than that in the sitting state. A study based on EEG showed that the theta activities in fronto-central and centro-parietal cortical areas significantly increased with increasing balance task demands. The dynamic reorganization of the cortical network can contribute to an optimization of balance control (Mierau et al., 2015, 2017). According to the $\mathrm{mCD}$ results among the brain regions, the causal influences from $\mathrm{OL}$ to $\mathrm{MC}$ and from PFC to OL were more dominant during standing than during sitting. Different postures lead to differences in hemodynamic responses in the cerebral and systemic vasculature, where autonomic reflexes are mediated by sympathetic, parasympathetic, and somatic sensory nerves (Olufsen et al., 2004). Pfeifer reported the decreased functions of sympathetic nervous system with aging (Pfeifer et al., 1983). The significant difference in EC between sitting and standing states in the elderly revealed that reorganization of the interregional interactions is required to maintain the performance in different posture states.

Compared with the young group in sitting state, the elderly group generally decreased directed interactions among the six brain regions. Our study revealed that the elderly exhibited significantly decreased regulating influence from PFC and $\mathrm{OL}$ to MC. Age-related decrease in EC across brain regions suggested decreased regulation on motor area in the elderly. This phenomenon may be due to the significantly attenuated function of neurovascular coupling control in PFC and OL with aging. This conclusion is supported by the finding that age-related shrinkage in gray matter volume and deterioration in cerebral white matter integrity are mainly distributed in frontal areas (Park and Reuterlorenz, 2009). These results may also be related to the connectivity between frontal-parietal areas, which are impaired in aging as shown from structural imaging (Burzynska et al., 2010; Meier-Ruge et al., 2010; Madden et al., 2012). A previous study reported that the disturbance and misperception of the body schema was caused by lesions in the OL (Coslett, 1998). Basing on our results, we suggest that the attenuated causal influence from OL to MC explains why elderly people fall easily. In interval II, the results in standing state showed significantly increased coupling strength from MC to PFC in elderly subjects as compared with that in the young group. That is, the elderly exerted great influence from MC to PFC during standing state. This result indicates that the elderly greatly relies on the PFC and its role in cognitive resources during standing state. This finding is consistent with the results of previous studies that additional cognitive resources need to be recruited as a measure of compensation to maintain performance with 


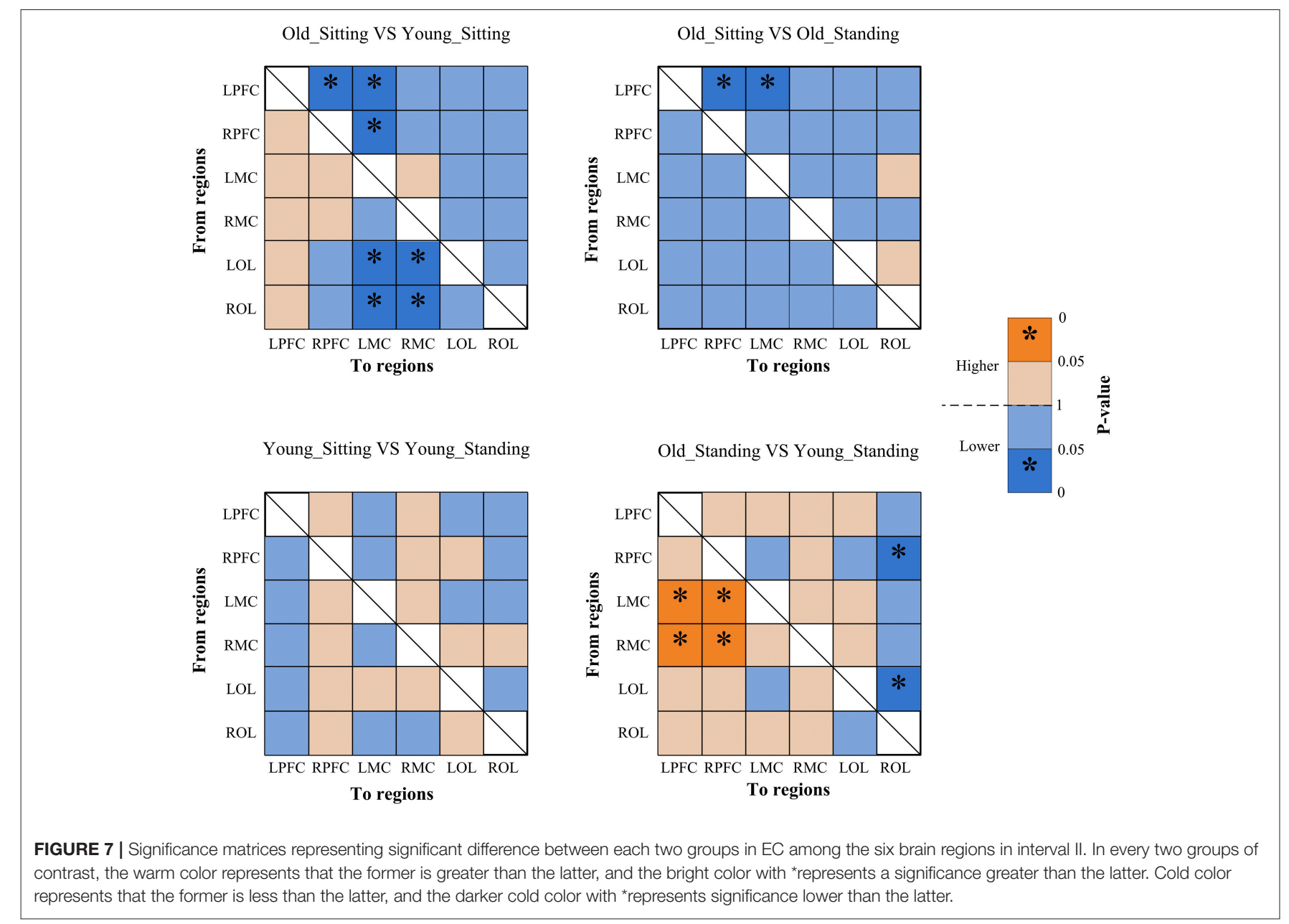

TABLE 2 | Correlations between interregional EC and cognitive performance.

\begin{tabular}{llllc}
\hline State & Interval & Interregional EC & \multicolumn{2}{c}{ MMSE scores } \\
\cline { 3 - 4 } & & & $\boldsymbol{R}$ & $\boldsymbol{P}$ \\
\hline \multirow{2}{*}{ Sitting } & $\mathrm{N}$ & 0.689 & $0.002^{*}$ \\
& $\mathrm{LPFC} \rightarrow$ RPFC & 0.679 & $0.003^{*}$ \\
& RPFC $\rightarrow$ LPFC & 0.487 & $0.047^{*}$ \\
& RPFC $\rightarrow$ LMC & 0.486 & $0.048^{*}$ \\
& LMC $\rightarrow$ RPFC & 0.531 & $0.028^{*}$ \\
& RPFC $\rightarrow$ RMC & 0.505 & $0.039^{*}$ \\
& RPFC $\rightarrow$ LOL & 0.489 & $0.047^{*}$ \\
& RPFC $\rightarrow$ ROL & &
\end{tabular}

${ }^{*}$ Denotes the significant correlations, $p<0.05$.

aging (Vandenbossche et al., 2011; Beurskens et al., 2014). By combining this finding and the $\mathrm{mCD}$ results across the brain network (Figure 5), we observed that the significantly increased causal interactions from MC to PFC in the elderly are a feedback effect. The $\mathrm{mCD}$ in this interval was approximately consistent in both groups. PFC played a predominant regulatory role on OL and $\mathrm{MC}$ and $\mathrm{MC}$ was also regulated by the OL.

The results also revealed the existence of age-related changes in EC in response to posture changes in interval I. The origin of the oscillations in interval I $(0.052-0.145 \mathrm{~Hz})$ is associated with vasomotor activities, thus contributing to the regulation of $\mathrm{rCBF}$ according to regional metabolic demands. The realization of brain function is critically dependent on a steady supply of blood (Girouard and Iadecola, 2006; Shiogai et al., 2010). With an increase or decrease in intravascular pressure, the vascular smooth muscles contract or relax to correspond with the blood oxygen concentration (Shiogai et al., 2010). The cerebral oscillations originated locally from intrinsic myogenic activity of smooth vascular muscle cells and were endowed with neural autonomic control of cerebral circulation to assure cerebrovascular function (Rowley et al., 2007; Shiogai et al., 2010). Under different conditions, myogenic regulatory mechanism plays a certain role in stabilizing CBF fluctuation. By comparing the effective interactions among the network in different posture states, we observed that the coupling strength from RPFC to MC in interval I significantly increased in the young group in response to posture changes. The elderly group exhibited no significant difference in EC during sitting and standing states. These results revealed that according to varying postures, different regional metabolic demands are required to meet stable CBF. This phenomenon may result in differences in the interaction among brain regions. The causal influence 
from $\mathrm{PFC}$ to $\mathrm{MC}$ in the young group was significantly stronger in the standing state than in the sitting state. These regions were assumed to play key roles in cognitive control function (Miller and Cohen, 2001) and coordination of sensory and motor functions (Basso et al., 2014). These results may be attributed to the strong myogenic regulation mechanism in the young group and result in prompt and reliable regulation of $\mathrm{rCBF}$ in response to posture changes. The absence of significant difference in response to posture change in the elderly may be due to the increased vessel stiffness with aging and the myogenic regulation degeneration in the elderly group.

In the sitting state, the directed coupling strength between LPFC and RPFC was significantly lower in the elderly group compared with the young group, indicating that mutual causal interaction between them decreased with aging. Such decreased connectivity was significantly correlated with the MMSE scores in elderly, which indicated the cognitive performance. PFC plays a key role in complex cognitive behavior (Miller and Cohen, 2001). Based on the results of the positive relationship between the cognitive performance and causal interactions between left PFC and right PFC, we speculated that such decreased EC may be an indicator of cognitive decline. This finding was in line with the idea that the altered patterns of connectivity between PFC and other regions attributed to the impairment in cognitive control (Meunier et al., 2009). These results agree with those of previous studies, showing that frontal functions are susceptible to degenerative changes in normal aging (Park and Reuterlorenz, 2009; Antonenko and Flöel, 2013). These results may be attributed to reduced spontaneous activity in microvascular smooth muscle cells together with increased vessel stiffness with aging (Schroeter et al., 2004; Li et al., 2013a). Other notable findings in this interval were the agerelated changes in $\mathrm{mCD}$ among the brain regions. In the young group, PFC predominantly influenced MC and OL. However, in the elderly group, the $\mathrm{mCD}$ results showed that the $\mathrm{MC}$ predominantly influenced PFC and OL, implying that MC played a main regulatory role in interval I. By combing present and previous results on coupling strength, we revealed the impaired prefrontal regulation in the elderly and the theory that longranged regulatory mechanisms become vulnerable with aging (Dardo and Volkow, 2012). In the elderly group, the regulating ability of smooth muscle cells in MC was preserved or enhanced in interval I. The brain possibly reorganizes the regulation ability of smooth muscle cells to adapt to decreased cognitive deficits with age.

\section{LIMITATIONS}

One consideration of the results is that systemic activity interferences in this interval must be further addressed. In our

\section{REFERENCES}

Andrewshanna, J. R., Snyder, A. Z., Vincent, J. L., Lustig, C., Head, D., Raichle, M. E., et al. (2007). Disruption of large-scale brain systems in advanced aging. Neuron 56, 924-935. doi: 10.1016/j.neuron.2007.10.038 study, the range of interval I was within $0.052-0$. $145 \mathrm{~Hz}$. We cannot fully exclude contamination by heart rate and blood pressure fluctuations. Cerebral oscillations in interval I were considered to originate locally from the intrinsic myogenic activity of smooth vascular muscle cells and partly under neural autonomic control of cerebral circulation (Rowley et al., 2007; Shiogai et al., 2010; Li et al., 2012). A study reported that in the frequency interval $0.04-0.15 \mathrm{~Hz}$, the contribution of systemic signals to hemodynamic changes reached only $35 \%$ when carried by oxy-Hb (Katura et al., 2006). These systemic activity interferences may affect the results of EC. Thus, the interference of Mayer waves should be considered in future studies.

\section{CONCLUSIONS}

In this study, we revealed posture-related changes in frequencyspecific EC in elderly subjects based on dynamic Bayesian inference. The brain network features different regulatory modes at different frequencies, which change to maintain stable $\mathrm{CBF}$ in different posture states. The results reflect the age-related changes in effective interactions across brain regions in response to posture changes. Significantly decreased interregional EC was found in intervals I and II in elderly in the sitting state as compared with that in the young group. The decreased connectivity in interval I was also strongly positively correlated with cognitive performance in the elderly. Compared with that in the young group, the influence from MC to PFC significantly increased in intervals II in the elderly group in standing state, suggesting that the elderly subjects greatly rely on cognitive resources as a compensation to maintain performance in aging. These findings may provide an evidence for cognitive decline and can aid in detecting the functional performance of neurovascular coupling mechanism in the elderly.

\section{AUTHOR CONTRIBUTIONS}

ZL: designed the study and edited the manuscript; $\mathrm{CH}$ : did the experiment, analyzed the data and drafted the manuscript; LB: did the experiment; GX: analyzed the data; YL: performed the statistical analysis; MZ: contributed to the physiological interpretation of the results; LS: edited the manuscript.

\section{ACKNOWLEDGMENTS}

This work was supported by the National Natural Science Foundation of China (grant numbers 31771071, 61761166007, 11732015); the Fundamental Research Funds for Central Public Welfare Research Institutes (118009001000160001).

Antonenko, D., and Flöel, A. (2013). Healthy aging by staying selectively connected: a mini-review. Gerontology 60, 3-9. doi: 10.1159/000354376

Astafiev, S. V., Stanley, C. M., Shulman, G. L., and Corbetta, M. (2004). Extrastriate body area in human occipital cortex responds to the performance of motor actions. Nat. Neurosci. 7, 542-548. doi: 10.1038/nn1241 
Basso, M. S., Bisconti, S., Muthalib, M., Spezialetti, M., Cutini, S., Ferrari, M., et al. (2014). A semi-immersive virtual reality incremental swing balance task activates prefrontal cortex: a functional near-infrared spectroscopy study. Neuroimage 85(Pt 1), 451-460. doi: 10.1016/j.neuroimage.2013.05.031

Beek, A. H. V., Claassen, J. A., Rikkert, M. G. O., and Jansen, R. W. (2008). Cerebral autoregulation: an overview of current concepts and methodology with special focus on the elderly. J. Cereb. Blood Flow Metab. 28, 1071-1085. doi: $10.1038 / j \mathrm{cbfm} .2008 .13$

Bernjak, A., Stefanovska, A., McClintock, P. V. E., Owen-Lynch, P. J., and Clarkson, P. B. M. (2012). Coherence between fluctuations in blood flow and oxygen saturation. Fluct. Noise Lett. 11:1240013. doi: 10.1142/S0219477512400135

Beurskens, R., Helmich, I., Rein, R., and Bock, O. (2014). Age-related changes in prefrontal activity during walking in dual-task situations: a fNIRS study. Int. J. Psychophysiol. 92, 122-128. doi: 10.1016/j.ijpsycho.2014.03.005

Biswal, B., Yetkin, F. Z., Haughton, V. M., and Hyde, J. S. (2010). Functional connectivity in the motor cortex of resting human brain using echoplanar MRI. Magn. Reson. Med. 34, 537-541. doi: 10.1002/mrm.19103 40409

Bu, L., Li, J., Li, F., Liu, H., and Li, Z. (2016). Wavelet coherence analysis of cerebral oxygenation signals measured by near-infrared spectroscopy in sailors: an exploratory, experimental study. BMJ Open 6:e013357. doi: 10.1136/bmjopen-2016-013357

Burzynska, A. Z., Preuschhof, C., Bäckman, L., Nyberg, L., Li, S. C., Lindenberger, U., et al. (2010). Age-related differences in white matter microstructure: region-specific patterns of diffusivity. Neuroimage 49, 2104-2112. doi: 10.1016/j.neuroimage.2009.09.041

Corbetta, M., Miezin, F. M., Dobmeyer, S., Shulman, G. L., and Petersen, S. E. (1991). Selective and divided attention during visual discriminations of shape, color, and speed: functional anatomy by positron emission tomography. $J$. Neurosci. 11, 2383-2402.

Coslett, H. B. (1998). Evidence for a disturbance of the body schema in neglect. Brain Cogn. 37, 527-544. doi: 10.1006/brcg.1998.1011

Dardo, T., and Volkow, N. D. (2012). Aging and functional brain networks. Mol. Psychiatry 17, 471, 549-558. doi: 10.1038/mp.2011.81

Fabiani, M., Gordon, B. A., Maclin, E. L., Pearson, M. A., Brumbackpeltz, C. R., Low, K. A., et al. (2014). Neurovascular coupling in normal aging: a combined optical, ERP and fMRI study. Neuroimage 85(Pt 1), 592-607. doi: 10.1016/j.neuroimage.2013.04.113

Friston, K. J. (1994). Functional and effective connectivity in neuroimaging: a synthesis. Hum. Brain Mapp. 2, 56-78. doi: 10.1002/hbm.460020107

Friston, K. J. (2011). Functional and effective connectivity: a review. Brain Connect. 1, 13-36. doi: 10.1089/brain.2011.0008

Girouard, H., and Iadecola, C. (2006). Neurovascular coupling in the normal brain and in hypertension, stroke, and Alzheimer disease. J. Appl. Physiol. 100, 328-335. doi: 10.1152/japplphysiol.00966.2005

Han, Q., Zhang, M., Li, W., Gao, Y., Xin, Q., Wang, Y., et al. (2014). Wavelet coherence analysis of prefrontal tissue oxyhaemoglobin signals as measured using near-infrared spectroscopy in elderly subjects with cerebral infarction. Microvasc. Res. 95, 108-115. doi: 10.1016/j.mvr.2014.08.001

Hedden, T., and Gabrieli, J. D. E. (2004). Insights into the ageing mind: a view from cognitive neuroscience. Nat. Rev. Neurosci. 5, 87-96. doi: 10.1038/nr n1323

Hogenhout, M. (2013). The age-related regulation of sensorimotor integration in human postural control. Mol. Cell. Biol. 8, 4889-4895. doi: $10.1152 /$ jn. 00516.2003

Hotta, H. (2016). Neurogenic control of parenchymal arterioles in the cerebral cortex. Prog. Brain Res. 225, 3-39. doi: 10.1016/bs.pbr.2016.03.001

Jones, W. J., Williams, L. S., Bruno, A., and Biller, J. (2003). Hypertension and cerebrovascular disease. Seminars Cerebrovasc. Dis. Stroke 3, 144-154. doi: 10.1053/j.scds.2003.00.029

Kamran, M. A., Yung, J. M., and Mannan, M. M. N. (2015). Optimal hemodynamic response model for functional near-infrared spectroscopy. Front. Behav. Neurosci. 9:151. doi: 10.3389/fnbeh.2015.00151

Katura, T., Tanaka, N., Obata, A., Sato, H., and Maki, A. (2006). Quantitative evaluation of interrelations between spontaneous low-frequency oscillations in cerebral hemodynamics and systemic cardiovascular dynamics. Neuroimage 31, 1592-1600. doi: 10.1016/j.neuroimage.2006.02.010
Kiyoka, K., Sophie, S., Monica, P., Marion, D., Carolin, J., Mohamed, D., et al. (2013). Aging-related episodic memory decline: are emotions the key? Front. Behav. Neurosci. 7:2. doi: 10.3389/fnbeh.2013.00002

Li, Z., Wang, Y., Li, Y., Wang, Y., Li, J., and Zhang, L. (2010). Wavelet analysis of cerebral oxygenation signal measured by near infrared spectroscopy in subjects with cerebral infarction. Microvasc. Res. 80, 142-147. doi: 10.1016/j.mvr.2010.02.004

Li, Z., Zhang, M., Xin, Q., Chen, G., Liu, F., and Li, J. (2012). Spectral analysis of near-infrared spectroscopy signals measured from prefrontal lobe in subjects at risk for stroke. Med. Phys. 39, 2179-2185. doi: 10.1118/1.3696363

Li, Z., Zhang, M., Xin, Q., Luo, S., Cui, R., Zhou, W., et al. (2013a). Age-related changes in spontaneous oscillations assessed by wavelet transform of cerebral oxygenation and arterial blood pressure signals. J. Cereb. Blood Flow Metab. 33, 692-699. doi: 10.1038/jcbfm.2013.4

Li, Z., Zhang, M., Xin, Q., Luo, S., Zhou, W., Cui, R., et al. (2013b). Assessment of cerebral oxygenation oscillations in subjects with hypertension. Microvasc. Res. 88(Suppl. C), 32-41. doi: 10.1016/j.mvr.2013.04.003

Madden, D. J., Bennett, I. J., Burzynska, A., Potter, G. G., Chen, N. K., and Song, A. W. (2012). Diffusion tensor imaging of cerebral white matter integrity in cognitive aging. BBA-Mol. Basis Dis. 1822, 386-400. doi: 10.1016/j.bbadis.2011.08.003

Meier-Ruge, W., Ulrich, J., Brühlmann, M., and Meier, E. (2010). Age-related white matter atrophy in the human brain. Ann. N.Y. Acad. Sci. 673, 260-269. doi: 10.1111/j.1749-6632.1992.tb27462.x

Meunier, D., Achard, S., Morcom, A., and Bullmore, E. (2009). Age-related changes in modular organization of human brain functional networks. Neuroimage 44, 715-723. doi: 10.1016/j.neuroimage.2008.09.062

Mierau, A., Hülsdünker, T., and Strüder, H. K. (2015). Changes in cortical activity associated with adaptive behavior during repeated balance perturbation of unpredictable timing. Front. Behav. Neurosci. 9:272. doi: 10.3389/fnbeh.2015.00272

Mierau, A., Pester, B., Hülsdünker, T., Schiecke, K., Strüder, H. K., and Witte, H. (2017). Cortical correlates of human balance control. Brain Topogr. 30, 434-446. doi: 10.1007/s10548-017-0567-x

Mihara, M., Miyai, I., Hatakenaka, M., Kubota, K., and Sakoda, S. (2008). Role of the prefrontal cortex in human balance control. Neuroimage 43, 329-336. doi: 10.1016/j.neuroimage.2008.07.029

Mitchell, A. J., Leon, K. J. B., and Reuber, M. (2010). The influence of cognitive impairment on health-related quality of life in neurological disease. Acta Neuropsychiatr. 22, 2-13. doi: 10.1111/j.1601-5215.2009.00439.x

Miller, E. K., and Cohen, J. D. (2001). An integrative theory of prefrontal cortex function. Annu. Rev. Neurosci. 24, 167-202. doi: 10.1146/annurev.neuro.24.1.167

Moran, J., and Desimone, R. (1985). Selective attention gates visual processing in the extrastriate cortex. Science 229, 782-784. doi: 10.1126/science.4023713

Naseer, N., and Hong, K. S. (2015). fNIRS-based brain-computer interfaces: a review. Front. Hum. Neurosci. 9:3. doi: 10.3389/fnhum.2015.00003

Noori, F. M., Naseer, N., Qureshi, N. K., Nazeer, H., and Khan, R. A. (2017). Optimal feature selection from fNIRS signals using genetic algorithms for BCI. Neurosci. Lett. 647, 61-66. doi: 10.1016/j.neulet.2017.03.013

Obrig, H., Neufang, M., Wenzel, R., Kohl, M., Steinbrink, J., Einhäupl, K., et al. (2000). Spontaneous low frequency oscillations of cerebral hemodynamics and metabolism in human adults. Neuroimage 12, 623-639. doi: 10.1006/nimg.2000.0657

Olufsen, M., Tran, H., and Ottesen, J. (2004). Modeling cerebral blood flow control during posture change from sitting to standing. Cardiovasc. Eng. Int. J. 4, 47-58. doi: 10.1023/B:CARE.0000025122.46013.1a

Orgs, G., Dovern, A., Hagura, N., Haggard, P., Fink, G. R., and Weiss, P. H. (2016). Constructing visual perception of body movement with the motor cortex. Cereb. Cortex 26, 440-449. doi: 10.1093/cercor/bhv262

Park, D. C., and Reuterlorenz, P. (2009). The adaptive brain: aging and neurocognitive scaffolding. Annu. Rev. Psychol. 60, 173-196. doi: 10.1146/annurev.psych.59.103006.093656

Park, H. J., and Friston, K. (2013). Structural and functional brain networks: from connections to cognition. Science 342:1238411. doi: 10.1126/science.1238411

Pfeifer, M. A., Weinberg, C. R., Cook, D., Best, J. D., Reenan, A., and Halter, J. B. (1983). Differential changes of autonomic nervous system function with age in man. Am. J. Med. 75, 249-258. doi: 10.1016/0002-9343(83)91201-9 
Rowley, A. B., Payne, S. J., Tachtsidis, I., Ebden, M. J., Whiteley, J. P., Gavaghan, D. J., et al. (2007). Synchronization between arterial blood pressure and cerebral oxyhaemoglobin concentration investigated by wavelet crosscorrelation. Physiol. Meas. 28, 161-173. doi: 10.1088/0967-3334/28/2/005

Sasai, S., Homae, F., Watanabe, H., Sasaki, A. T., Tanabe, H. C., Sadato, N., et al. (2012). A NIRS-fMRI study of resting state network. Neuroimage 63, 179-193. doi: 10.1016/j.neuroimage.2012.06.011

Scholkmann, F., Kleiser, S., Metz, A. J., Zimmermann, R., Mata, P. J., Wolf, U., et al. (2014). A review on continuous wave functional near-infrared spectroscopy and imaging instrumentation and methodology. Neuroimage 85(Pt 1), 6-27. doi: 10.1016/j.neuroimage.2013.05.004

Schroeter, M. L., Schmiedel, O., and von Cramon, D. Y. (2004). Spontaneous lowfrequency oscillations decline in the aging brain. J. Cereb. Blood Flow Metab. 24, 1183-1191. doi: 10.1097/01.WCB.0000135231.90164.40

Shiogai, Y., Stefanovska, A., and Mcclintock, P. V. E. (2010). Nonlinear dynamics of cardiovascular ageing. Phys. Rep. 488, 51-110. doi: 10.1016/j.physrep.2009.12.003

Sorond, F. A., Hurwitz, S., Salat, D. H., Greve, D. N., and Fisher, N. D. (2013). Neurovascular coupling, cerebral white matter integrity, and response to cocoa in older people. Neurology 81, 904-909. doi: 10.1212/WNL.0b013e3182 a351aa

Sorond, F. A., Kiely, D. K., Galica, A., Moscufo, N., Serrador, J. M., Iloputaife, I., et al. (2011). Neurovascular coupling is impaired in slow walkers: the MOBILIZE Boston Study. Ann. Neurol. 70, 213-220. doi: 10.1002/ana.22433

Sporns, O., and Kötter, R. (2004). Motifs in brain networks. PLoS Biol. 2:e369. doi: 10.1371/journal.pbio.0020369

Spreng, R. N., and Schacter, D. L. (2012). Default network modulation and largescale network interactivity in healthy young and old adults. Cereb. Cortex 22, 2610. doi: 10.1093/cercor/bhr339

Stankovski, T., Duggento, A., Mcclintock, P. V. E., and Stefanovska, A. (2014). A tutorial on time-evolving dynamical Bayesian inference. Eur. Phys. J. Spec. Top. 223, 2685-2703. doi: 10.1140/epist/e2014-02286-7

Stefanova, I., Stephan, T., Beckerbense, S., Dera, T., Brandt, T., and Dieterich, M. (2013). Age-related changes of blood-oxygen-level-dependent signal dynamics during optokinetic stimulation. Neurobiol. Aging 34, 2277. doi: 10.1016/j.neurobiolaging.2013.03.031

Tan, Q., Ming, Z., Yi, W., Zhang, M., Wang, B., Xin, Q., et al. (2016). Agerelated alterations in phase synchronization of oxyhemoglobin concentration changes in prefrontal tissues as measured by near-infrared spectroscopy signals. Microvasc. Res. 103, 19-25. doi: 10.1016/j.mvr.2015.10.002

Taniwaki, T., Okayama, A., Yoshiura, T., Togao, O., Nakamura, Y., Yamasaki, T., et al. (2007). Age-related alterations of the functional interactions within the basal ganglia and cerebellar motor loops in vivo. Neuroimage 36, 1263-1276. doi: 10.1016/j.neuroimage.2007.04.027
Teng, Y., Ding, H., Gong, Q., Jia, Z., and Huang, L. (2006). Monitoring cerebral oxygen saturation during cardiopulmonary bypass using near-infrared spectroscopy: the relationships with body temperature and perfusion rate. J. Biomed. Opt. 11, 9-0. doi: 10.1117/1.2187422

Tomislav, S., Spase, P., Johan, R., Smith, A. F., Mcclintock, P. V. E., and Aneta, S. (2016). Alterations in the coupling functions between cortical and cardiorespiratory oscillations due to anaesthesia with propofol and sevoflurane. Philos. Trans. A Math. Phys. Eng. Sci. 374:20150186. doi: 10.1098/rsta.2015.0186

Vandenbossche, J., Deroost, N., Soetens, E., Spildooren, J., Vercruysse, S Nieuwboer, A., et al. (2011). Freezing of gait in Parkinson disease is associated with impaired conflict resolution. Neurorehabil. Neural Repair 25, 765-773. doi: 10.1177/1545968311403493

Vermeij, A., As, M. D. A., Kessels, R. P., van Beek, A. H., and Claassen, J. A. (2014). Very-low-frequency oscillations of cerebral hemodynamics and blood pressure are affected by aging and cognitive load. Neuroimage 85(Pt 1), 608-615. doi: 10.1016/j.neuroimage.2013.04.107

Wang, B., Ming, Z., Bu, L., Xu, L., Wei, W., and Li, Z. (2016). Posturerelated changes in brain functional connectivity as assessed by wavelet phase coherence of NIRS signals in elderly subjects. Behav. Brain Res. 312, 238. doi: 10.1016/j.bbr.2016.06.037

Wen, W., Zhu, W., He, Y., Kochan, N. A., Reppermund, S., Slavin, M. J., et al. (2011). Discrete neuroanatomical networks are associated with specific cognitive abilities in old age. J. Neurosci. 31, 1204-1212. doi: 10.1523/JNEUROSCI.4085-10.2011

Xu, G., Zhang, M., Wang, Y., Liu, Z., Huo, C., Li, Z., et al. (2017). Functional connectivity analysis of distracted drivers based on the wavelet phase coherence of functional near-infrared spectroscopy signals. PLoS ONE 12:e0188329. doi: 10.1371/journal.pone.0188329

Zhang, R., Zuckerman, J. H., Iwasaki, K., Wilson, T. E., Crandall, C. G., and Levine, B. D. (2002). Autonomic neural control of dynamic cerebral autoregulation in humans. Circulation 106, 1814-1820. doi: 10.1161/01.CIR.0000031798.07790.FE

Conflict of Interest Statement: The authors declare that the research was conducted in the absence of any commercial or financial relationships that could be construed as a potential conflict of interest.

Copyright (๑) $2018 \mathrm{Huo}$, Zhang, Bu, Xu, Liu, Li and Sun. This is an open-access article distributed under the terms of the Creative Commons Attribution License (CC $B Y)$. The use, distribution or reproduction in other forums is permitted, provided the original author(s) and the copyright owner are credited and that the original publication in this journal is cited, in accordance with accepted academic practice. No use, distribution or reproduction is permitted which does not comply with these terms. 